\title{
Instrument implementation of an automated complex for recording physiological parameters of drivers
}

\author{
Robert Khizbullin ${ }^{1, *}$, Radik Khizbullin ${ }^{1}$, and Valerii Galiakhmetov ${ }^{1}$ \\ ${ }^{1}$ Kazan State Power Engineering University, Krasnoselskaya, 51, Kazan, 420066, Russia
}

\begin{abstract}
Goal of research: creation of an automated complex for quick and effective identification of an unacceptable condition of drivers before going to run and predicting their physiological state during a work shift. To achieve this goal in the creation of devices for the automated complex the following methods were used: photoplethysmographic method, pulse oximetric method, thermometric method. In the course of the research, results were achieved that make it possible to record physiological parameters of drivers with high reliability. This is achieved thanks to the use of the "isobestic point" photoplethysmogram in the registration at a length of $805 \mathrm{~nm}$, the use of a two-channel pulse oximeter at wavelengths of $660 \mathrm{~nm}$ and $910 \mathrm{~nm}$.
\end{abstract}

\section{Introduction}

Road safety affects almost any citizen, because one way or another we are all either road users - drivers, pedestrians or just passengers. But, as passengers of public transport, people give the driver or driver not only their health, but even their lives. Since accidents with vehicles carrying a large number of people are particularly tragic, it is precisely the fault of drivers, passengers must be sure of the safety of their trip. The driver, like any other person, is prone to diseases and psychophysical disorders. It follows that one of the fundamental factors for ensuring road safety is the state of health of the driver. High traffic intensity due to a significant increase in the number of vehicles places higher demands on drivers in terms of health status. Therefore, an important task facing public and city transport companies that carry out transportation of people and are licensed to conduct medical examinations [1] is to reliably identify deviations in the health status of drivers and to conduct quality pre-trip and post-trip examinations with further monitoring of the physical and psycho-physiological state of drivers and train drivers.

Medical examination of vehicle drivers is one of the most important measures aimed at ensuring road safety. The current legislation provides for three types of medical examinations of vehicle drivers: pre-trip, post-trip and current (Article 23 of the Federal Law "On Road Traffic Safety" N 169-FL) [2].

\footnotetext{
*Corresponding author: Robert.Khizbullin@mail.ru
} 
The obligation to conduct both pre-trip and post-trip medical examinations is also confirmed in the order of the Ministry of Transport of Russia dated 18.09.2008 N 152 [3], and other normative acts: paragraph 5.2.54 the procedure for conducting pre-shift, pre-trip, post-shift, post-trip medical examinations (Provisions On the Ministry of Health of the Russian Federation, approved by Decree of the Government of the Russian Federation of June 19, 2012 N. 608) [4], clause 7 of article of the Federal Law of November 21, 2011 N. 323-FL "On the Basics of Protecting the Health of Citizens in the Russian Federation"[5].

The Ministry of Health of Russia approved the procedure for conducting pre-shift, pretrip and post-shift, post-trip medical examinations of certain categories of workers, issued an order dated December 15, 2014 N. 835n "On approval of the procedure for conducting pre-shift, pre-trip and post-shift, post-trip medical examinations" [6], which was registered by the Ministry of Justice of Russia on 16.04 .2015 and entered into force on May 1, 2015.

This regulatory act [6] defines the rules for pre-shift, pre-trip and post-shift, post-trip medical examinations, clause 4: "Pre-shift (pre-trip) medical examinations are carried out before the start of a work shift (flight) in order to identify signs of exposure to harmful and (or) hazardous production "factors, conditions and diseases that impede the performance of work duties, including alcohol, drug or other toxic intoxication and the residual effects of such intoxication."

Drivers, machinists who successfully passed the pre-trip inspection at the beginning of the shift and have access to work cannot be isolated from their physiological state, i.e. their state of health during a work shift or trip.

In this regard, there is an urgent question about the medical and technical support of pre-trip and post-trip examinations, which are carried out before the start of the work shift, flight and after the shift, flight [6]. Thus, it is necessary to quickly and accurately determine the health status of drivers in a fairly short period of time.

\section{The solution of the current medical and technical problems and practical implementation}

To help doctors who monitor the physical condition of persons allowed to work, an automated medical hardware complex has been developed for pre-trip and post-trip examination of drivers and drivers. This computerized hardware complex (Fig. 1) consists of the following main functional hardware parts: a medical digital precision thermometer, a two-channel photoplethysmograph, and a three-channel laser pulse oximeter.

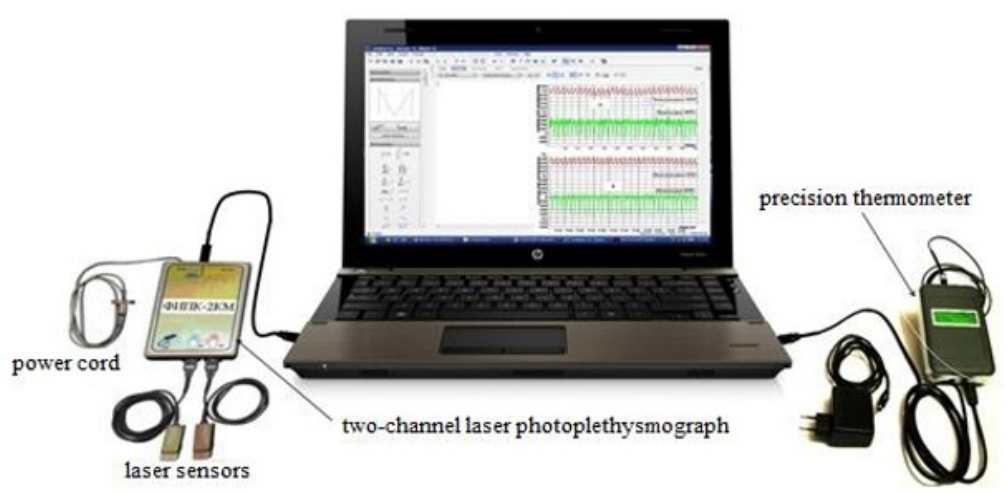

Fig. 1. Computerized hardware complex. 
The main objective of the developed automated medical hardware complex for pre-trip and post-trip examinations (examination) is to identify with high reliability the physiological state of the driver, as well as the predictability of his condition for the period of the work shift. In this case, the medical worker must quickly and accurately establish deviations from the physiological norms of the employee, make a conclusion about his admission to work. All data during each examination are recorded in the database of pretrip and post-trip inspections stored in the computer. As mentioned above, in the regulatory document (p. 10 [6], the mandatory procedures for inspection are taking readings of temperature, pressure, heart rate. automated complex, the measurement of temperature, pressure, heart rate can be carried out simultaneously, which reduces the examination time.

All data during each examination are recorded in the database of pre-trip and post-trip inspections stored in the computer. As mentioned above, in the regulatory document (p. 10 [6], the mandatory procedures for inspection are taking readings of temperature, pressure, heart rate. automated complex, the measurement of temperature, pressure, heart rate can be carried out simultaneously, which reduces the examination time.

As applied to the human body, the required temperature range of measurements is small: $+5 \ldots+50^{\circ} \mathrm{C}$. The accuracy of the temperature measurement should correspond to the temperature spread in the ranges of the norms of the relevant medical indicators. With regard to human research, accuracy within $\pm 0.05 \ldots \pm 0.1^{\circ} \mathrm{C}$ is quite sufficient. For a highquality visual representation of temperature measurements, and for relative measurements, as well as for graphical display of temperature changes, it is important to have high linearity and resolution of the measuring technical system. Temperature sensitivity should not exceed $0.01^{\circ} \mathrm{C}$.

Qualitative registration of the process of temperature changes in the body can only be provided by a low-inertia system, i.e. one whose inertness is at least an order of magnitude less than the temperature inertness of the measured area of the body. From this it follows that the temperature sensor should have the smallest overall dimensions and thermal inertia.

As sensors that allow temperature to be recorded with an accuracy of $\pm 0.1^{\circ} \mathrm{C}$ and having the smallest overall dimensions at the highest temperature sensitivity in the range + $5 \ldots+50{ }^{\circ} \mathrm{C}$, thermistors are most widely used in medicine [7].

The general technical requirements for medical thermometers [8-12] are fully met by the digital precision thermometer described in $[13,16,17]$ :

- range of the measured temperature $+5 \ldots+50^{\circ} \mathrm{C}$;

-relative error of the device when measuring temperature $0.1 \%$;

- type of temperature sensor used - thermistor;

- the absolute error of temperature measurement with a non-calibrated thermistor in the range $+25 \ldots+50 \circ \mathrm{C}$ is $\pm 0.1{ }^{\circ} \mathrm{C}$ (additional calibration is possible); - measurement rate - 15 values per second.

The developed digital medical precision thermometer (DMPT), which is part of an automated complex, consists of two main parts: a temperature registration unit and a temperature sensor, which has an external sensitive element (temperature sensor) -the thermistor. This temperature sensor allows you to record the temperature with an accuracy of $\pm 0.1^{\circ} \mathrm{C}$.

The length of the active part of the sensor is $450 \mathrm{~mm}$, the diameter is $1 \mathrm{~mm}$, the diameter of the thermistor head is $1.3 \mathrm{~mm}$. The sensor is connected to the unit using a $6.35 \mathrm{~mm}$ JACK connector. The total length of the sensor, including the lead-in cable and connector, is $1.5 \mathrm{~m}$. The sensor can be sterilized using any conventional hospital disinfectant, such as ethanol, isopropyl or chlorite compounds.

The registration unit processes the sensor signal and displays it on the display screen. Either a USB 2.0 interface or an external medical-grade power supply (a special 
adapter designed for constant voltage $+7.5 \ldots+12 \mathrm{~V}$ ) can be used as a power source for the main unit. When the unit is connected via USB 2.0, the temperature data is transferred to the computer and through specialized software in real time are displayed on the monitor, saved in the computer's memory as a simple text file.

The block diagram of the thermometer is shown in Fig. $2[14,16]$. The signal from the temperature sensor is fed to an amplifier, then to an analog-to-digital converter (ADC), where it is digitized and converted into a digital code, which is transmitted through a galvanic isolation to the microcontroller. The microcontroller processes the received data, calculating the temperature value, displays the received information on the display and transmits the temperature data in a digital code via a galvanically isolated USB 2.0 interface to the computer. A power selector is also included in the circuit.

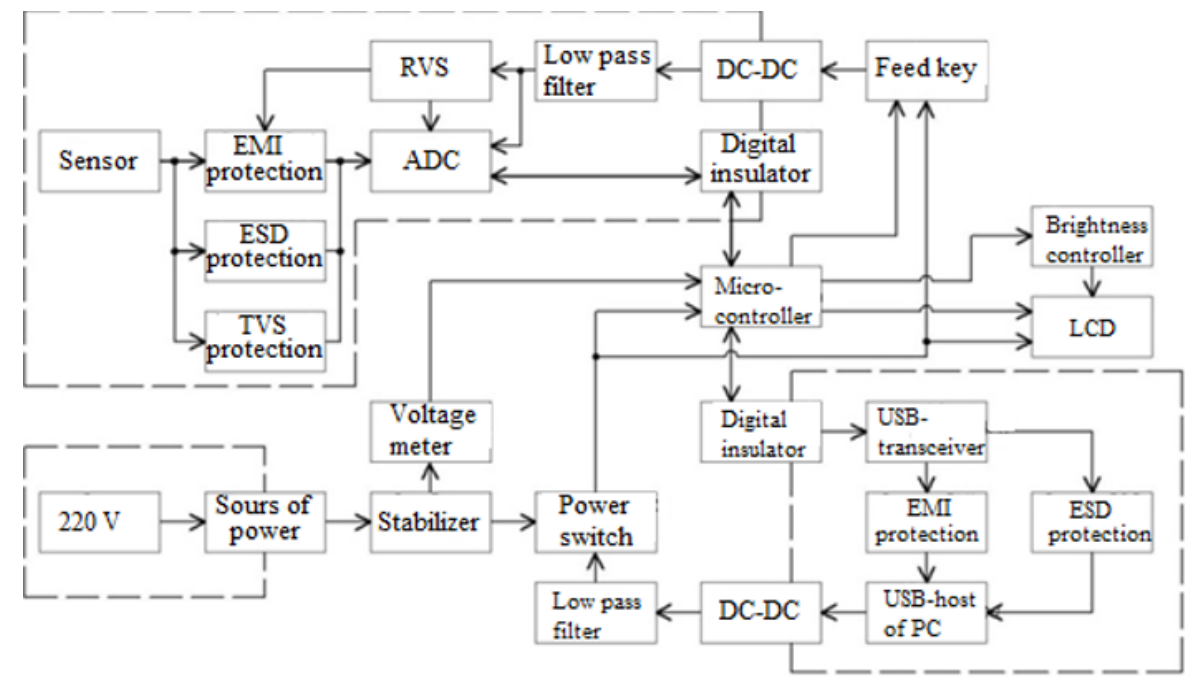

Fig. 2. Structural diagram of thermometer DMP.

The second instrument of the automated complex is a two-channel photoplethysmograph using the finger photoplethysmography method.

The use of digital photoplethysmography is of great diagnostic value in the quick and accurate assessment of local capillary blood flow, measurement of arterial and venous pressure, volume pulse, blood flow velocity, vascular tone, heart rate, minute and systolic blood volume. One of the features of the diagnostic capabilities of photoplethysmography is the ability to prevent negative reactions as a result of various physical factors, or squeezing blood vessels with a relatively stationary position of the driver's body while driving and identifying stressful conditions. The fact that digital photoplethysmography provides accurate and objective information on changes in blood circulation parameters when exposed to various physical and psychophysical factors over a short period of time allows it to be effectively used in assessing the physiological state of technical personnel and workers at production facilities in the energy sector where pathogenic factors are present affecting the human's healph. The diagnostic capabilities of photoplethism make it possible to predict the permissible dose of a pathogenic factor of exposure and to prevent negative reactions as a result of exposure to the body of this pathogenic factor.

The photoplethysmographic signal is associated with the hemodynamics of the whole organism, because reflects the form of the pulse wave, and the relationship with hemodynamics is not direct, i.e. The photoplethysmographic signal is the result of the action of a "low-pass filter" on the pulse wave. From this it follows that the analysis of the shape of the variable component of the signal can provide information about both local 
blood circulation and the hemodynamics of the body as a whole. That is why photoplethysmographs have recently been increasingly used in medical diagnostics.

At the time of the development of the two-channel photoplethysmograph, there were publications on the medical use of single-channel finger LED $(\lambda=940 \pm 20 \mathrm{~nm})$ photoplethysmography. The use of a single photosensor (one channel) with a wavelength of $920 \ldots 960 \mathrm{~nm}$ will inevitably lead to "physiological interference" when registering a PPG signal due to different amounts of light absorption by the main informative components of the blood flow - hydroxy and deoxyhemoglobin. Therefore, to obtain the necessary accuracy of less than 5\%, the repeatability and adequacy of the PPG signal is impossible. Therefore, a new laser $(\lambda=805 \pm 0.75 \mathrm{~nm}, \mathrm{P}=0.1 \ldots 0.2 \mathrm{~mW})$ two-channel two-finger photoplethysmograph was developed. The radiation wavelength of $804.25 \ldots 805.75 \mathrm{~nm}$ is the "isobetic point" for oxy- and deoxyhemoglobin, that is, this is the wavelength at which the spectral characteristics of these two substances coincide. Therefore, it is possible to avoid "physiological interference" in obtaining a photoplethysmogram.

An accurate assessment of the condition of the test person is not possible without taking into account his individual condition. In the analysis of existing photoplethysmographs, the individual state of the examined individuals is ignored when they are exposed to an external "energy" load. Physical factors such as electromagnetic radiation, radioactive radiation, electrical, magnetic, mechanical and other factors are susceptible to the human body and seriously affect the physiological state of the person.

Photoplethysmograms of different people can have a strong scatter in the magnitude of the constant component, which is associated with different optical densities of human tissues. To prevent saturation of the photo amplifier of the device, it is necessary to adjust either the gain or the current value of the illumination of the LED or laser. The device must necessarily have the function of adjusting one of these values. Otherwise, for some individuals, the photoplethysmogram signal can hardly be recorded without loss of information and severe distortion.

The requirements for quick diagnosis of the examined personnel are satisfied by the developed two-channel photoplethysmograph. The device also allows for artificial occlusion through an ordinary cuff. For this, the device has a valve and a compressor with a pressure sensor. Occlusive photoplethysmography is widely used in the diagnosis of vascular tone.

The main technical characteristics of the FPG-2KL device $[15,16,17]$ :

- registration, display and linear filtering of biomedical information in real time;

- support for standard (Nell-Cor-compatible) and laser photoplethysmographic sensors;

- synchronous registration of photoplethysmographic signal and pressure;

- the use of laser diodes to operate the device at the isobestic point $(805 \mathrm{~nm})$;

- a wide dynamic range (the photocurrent conversion coefficient varies in the range $2 \cdot 10^{5} \ldots 2 \cdot 10^{6}$ ), as a result of which the device can be used to study peripheral blood flow in both adults and children;

- two-channel operation mode. Both channels operate in parallel with a sampling frequency of $350 \mathrm{~Hz}$ per channel;

- band of the useful signal $0.1 \ldots 40 \mathrm{~Hz}$;

- automatic artificial occlusion using a compressor and electronic valve;

- option to control the level of artificial occlusion in accordance with the measurement procedure;

- bundled software for automated processing and analysis of registered information.

The use of a two-channel photoplethysmogram registration system makes it possible to compare the signals of both channels and, on the basis of strong differences between them, to reveal the fact that the subject is moving, and the corresponding data recorded during the movement should not be taken into account in further analysis. Thus, the signal quality and 
the adequacy of the automated diagnostic procedure significantly increase, the scope of photoplethysmographs significantly expands.

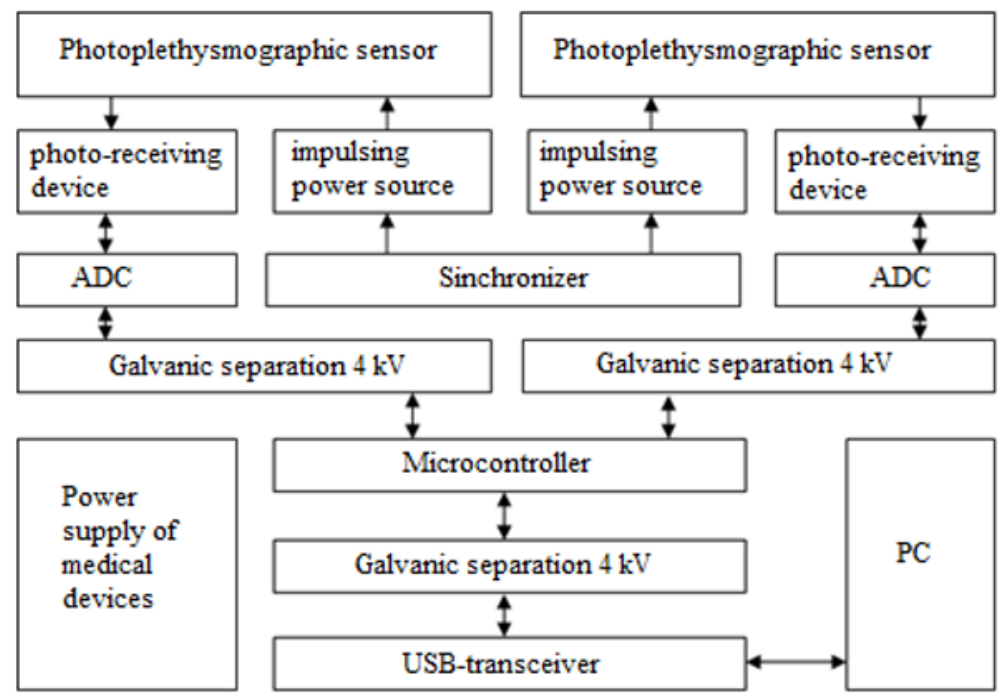

Fig. 3. Block diagram of a two-channel two-finger laser photoplethysmograph.

The third device in the automated medical complex is a laser pulse oximeter. A pulse oximeter is a device designed to measure the concentration of blood oxyhemoglobin in a pulsating vessel. The principle of operation of the device is based on the difference in extinction coefficients for oxyhemoglobin and deoxyhemoglobin at different wavelengths of the optical radiation. When using a laser diode as a radiation source, instead of an LED, it is possible to increase the quality of the device (reduce the signal-to-noise ratio of the recorded signal), expand the device's ability to work in people with weakened blood circulation (for example, with mildly pronounced vegetative-vascular dystonia), and increase the accuracy of measuring blood oxyhemoglobin concentration due to more narrow-band light sources, expand the possibilities of signal registration from vessels deeply located under the skin (for example, full thumbs). Moreover, the used laser pulse oximeter uses an additional infrared diode to increase the accuracy of measuring the concentration of oxyhemoglobin, as well as to determine the concentration of carboxyhemoglobin, which is formed in large quantities by inhalation of carbon monoxide. An increase in the concentration of carboxyhemoglobin leads to increased driver fatigue, a decrease in attention and reaction, up to a fainting state, therefore, monitoring the level of blood carboxyhemoglobin concentration before and after the shift should be performed without fail.

\section{Conclusions}

Ensuring road safety is one of the priority tasks of the state. An important role in solving this problem is assigned to medical personnel. They are entrusted with the duty of allowing citizens to drive vehicles. One of the forms of such admission is pre-trip and post-trip medical examinations of drivers. Timely identification of violations and deviations in the state of health of drivers is possible only with regular medical examinations. The proper organization and technical equipment of modern medical devices and complexes for pretrip and post-trip medical examinations is one of the key links in the prevention of road traffic accidents. 
The presented automated medical hardware complex for pre-trip and post-trip examination of drivers and drivers of transport enterprises is an effective tool for medical workers, which allows not only to significantly reduce the time required for pre-trip and post-trip examinations, but also to increase the efficiency of diagnosing deviations in the state of health of subjects Using this medical complex during examinations, the medical worker is guaranteed to receive high accuracy of measurements of such important physiological parameters of a person, such as temperature, pressure, pulse, and a number of other physiological parameters that can be diagnosed with these devices. The reliability and accuracy of the readings of the apparatus come from the selected methodology and principles of constructing apparatuses based on modern knowledge of the interaction of laser radiation with the main components of the blood flow.

The digital precision thermometer, which is part of the automated complex, allows you to solve an important medical problem - measuring the temperature of people with high accuracy and high speed. The features of the developed TMPP are: obtaining high accuracy characteristics, the temperature can be measured with a sensor with high accuracy and a resolution of $0.01^{\circ} \mathrm{C}$, which ensures registration of even insignificant temperature measurements for diagnostic purposes and significantly expands the field of medical thermometry, has automatic registration and recording of readings on a computer.

Advantages of the photoplethysmography method implemented in the developed FPG2KL photoplethysmograph:

1. provides registration of the reactions of the human body to an external physical effect of a pathogenic nature;

2. the use of a laser with a radiation wavelength of $805 \mathrm{~nm}$ eliminates the possibility of "physiological interference" associated with a change in the ratio of oxy to deoxyhemoglobin, since the spectral characteristics of the optical absorption of both types of hemoglobin intersect at an "isobestic point" at a wavelength of $805 \mathrm{~nm}$;

3. The use of a two-channel laser photoplethysmographic system for recording photoplethysmogram allows you to: compare both signals; to improve the overall quality of the PPG signal; expand the scope of photoplethysmographs.

4. the use of a two-channel laser photoplethysmograph as a recorder of changes in the state of the body, which is affected by pathogenic external influences (exhaust gases, etc.);

5. photoplethysmogram in this aspect reflects the dynamics of blood supply in the body. By registering such changes, it is possible to evaluate the effect of external pathogenic effects and further control the amount of such exposure in order to prevent negative consequences for the body as a whole.

6. The laser pulse oximeter has the functionality to determine the concentration of blood carboxyhemoglobin.

\section{References}

1. Decree of the Government of the Russian Federation of April 16, 2012 N 291 "On licensing of medical activities"

2. Federal law dated 10.12.2014 N 169-FZ (as amended on 07/13/2015) "On road safety"

3. Order of the Ministry of Transport of the Russian Federation of September 18, 2018, No. 152

4. Clause 5.2.54 of the Regulation on the Ministry of Health of the Russian Federation, approved by Decree of the Government of the Russian Federation of June 19, 2012, No. 608

5. Federal Law of November 21, 2011, No. 323

6. Order of the Ministry of Health of Russia dated 15.12.2014, No. 835 
7. R.G. Jackson, World of Electronics, 133-138 (2007)

8. J.M. Zurbuchen, Precision thermometry. Measurement science conference tutorial "Thermometry -fundamental and practice" (2000)

9. B.H. Childs, Practical Temperature Measurement (Oxford, 2001)

10. J.V. Nicholas, D.R. White, Traceable Temperature (2nd ed. Chichester, John Wiley\&Sons, LTD, 2001)

11. GOST R 50267.0.3-99, Medical electrical products

12. GOST R15.013-94, Medical devices

13. A.I. Laryushin, M.A. Galkin, et al., World of measurements, N 3, 21-25 (2010)

14. A.I. Laryushin, M.A. Galkin, et al., World of measurements, N 9, 9-17 (2010)

15. A.I. Laryushin, M.A. Galkin, et al., World of measurements, N 7, 22-28 (2010)

16. R.N. Khizbullin, A.I. Laryushin, News of higher educational institutions "Problems of Energy", N 1-2, 125-133 (2014)

17. Rob. Hizbullin, Rad. Hizbullin, E3S Web of Conferences, 124, 05037 (2019)

18. N.F. Kashapov, L.S. Sabitov, et al., IOP Conference Series: Materials Science and Engineering, 570(1), 012041

19. N.F. Kashapov, L.S. Sabitov, A.E. Auhadeev, et al., IOP Conference Series: Materials Science and Engineering, 570(1), 012040

20. E.M. Khusnutdinova, G.R. Khamidullina, et al. IOP Conference Series: Materials Science and Engineering, 412(1),012047 